\title{
Uni-DUE Student Team: Tackling fact checking through decomposable attention neural network
}

\author{
Jan Kowollik \\ University of Duisburg-Essen \\ jan.kowollik@stud.uni-due.de
}

\author{
Ahmet Aker \\ University of Duisburg-Essen \\ a.aker@is.inf.uni-due.de
}

\begin{abstract}
In this paper we present our system for the FEVER Challenge. The task of this challenge is to verify claims by extracting information from Wikipedia. Our system has two parts. In the first part it performs a search for candidate sentences by treating the claims as query. In the second part it filters out noise from these candidates and uses the remaining ones to decide whether they support or refute or entail not enough information to verify the claim. We show that this system achieves a FEVER score of 0.3927 on the FEVER shared task development data set which is a $25.5 \%$ improvement over the baseline score.
\end{abstract}

\section{Introduction}

In this paper we present our system for the FEVER Challenge ${ }^{1}$. The FEVER Challenge is a shared task on fact extraction and claim verification. Initially Thorne et al. (2018) created an annotated corpus of 185,445 claims and proposed a baseline system to predict the correct labels as well as the pieces of evidence for the claims.

Our system consist of two parts. In the first part we retrieve sentences that are relevant to a claim. The claim is used as query and is submitted to Lucene search API. The sentences found are candidates for pieces of evidence for the claim. Next in the second part we run a modified version of the Decomposable Attention network (Parikh et al., 2016) to predict the textual entailment between a claim and the candidate sentences found through searching but also between claim and all candidate sentences merged into one long text. This step gives us entailment probabilities. We also use a point system to filter out some noise (irrelevant sentences). Based on the remaining candidates we perform label prediction, i.e. whether the claim is supported, refuted or there is not enough evidence. Our system achieves a FEVER score of 0.3927 on the FEVER shared task development data set which is a $25.5 \%$ improvement over the baseline score.

\section{Data}

The data consists of two parts: the FEVER data set and the Wikipedia dump (Thorne et al., 2018).

The Wikipedia dump contains over five million pages but for each page only the first section was taken. The text on each page was split into sentences and each sentence was assigned an index. The page title is written using underscores between the individual words instead of spaces.

The FEVER data set contains the annotated claims that should be correctly predicted by the developed system. Each claim is annotated with one of the three labels SUPPORTS (verifiably true), REFUTES (verifiably false) and NOT ENOUGH INFO (not verifiable). For claims with the first two labels the pieces of evidence are provided as a combination of Wikipedia page title and sentence index on that page.

The FEVER data set created by Thorne et al. (2018) is split into a training set with 145,449 , a development set with 19,998 and a test set with 19, 998 annotated claims. The development and test sets are balanced while the training set has an approximately 16:6:7 split on the three labels. Each data set and also the Wikipedia dump is available at the FEVER web page ${ }^{2}$.

\section{System}

We decided to adopt the general two part structure of the baseline for our system with a key difference. The first part takes the claim and finds candidate sentences that ideally have a high chance of

\footnotetext{
${ }^{2}$ http://fever.ai/data.html
} 
being evidence for the claim. The second part determines the label and selects evidence sentences.

The baseline system uses the sentences found in the first part directly as evidence. In our system we only find candidate sentences in the first part and select the actual evidence sentences at the end of the second part. This allows us to operate on a larger number of sentences in the second part of the system and achieve higher recall.

\subsection{Finding Candidate Sentences}

The main idea of the first part of our system is to mimic human behavior when verifying a claim. If we take a claim about a person as an example, a human is likely to just take few keywords such as the person's name and use this to search for the right Wikipedia page to find evidence. We mimic this behavior by first extracting few keywords from the claim and use them to find candidate sentences in the Wikipedia dump.

\section{Extracting Keywords}

We use Named Entity Recognition (NER), Constituency Parsing and Dependency Parsing to extract keywords from each claim. For NER we use the neural network model created by Peters et al. (2018). We use all found named entities as keywords. For the Constituency Parsing we use the neural network model created by Stern et al. (2017). We extract all NP tagged phrases from the first two recursion layers as keywords because we found that this finds mostly subjects and objects of a sentence. These two neural networks both use the AllenNLP library (Gardner et al., 2018). For the dependency parsing we use the Standford Dependency Parser (Chen and Manning, 2014). We extract all subject and object phrases as keywords.

The NE recognition is our main source for keywords extraction while the other two systems provide additional keywords that either have not been found by the NER or that are not named entities in the first place. Example of the keywords being extracted from claims shown in Table 2 are shown in Table 1.

\section{Indexing the Wikipedia Dump}

After extracting the keywords we use the Lucene search $\mathrm{API}^{3}$ to find candidate sentences for each claim. Before searching with Lucene the Wikipedia texts need to be indexed. We treat each sentence as a separate document and index it. We

\footnotetext{
${ }^{3}$ https://lucene. apache.org/core/
}

exclude sentences that are empty and also those that are longer than 2000 characters.

For each sentence we also add the Wikipedia page title and make it searchable. For the title we replace all underscores with spaces to improve matching. In each sentence we replace the words $H e$, She, It and They with the Wikipedia page title that the sentence was found in. When looking at an entire Wikipedia page it is obvious who or what these words refer to but when searching individual sentences we do not have the necessary context available. We perform this replacement to provide more context.

\section{Searching for the Candidate Sentences}

We use three types of queries to search for candidate sentences for a claim:

- Type 1: For each keyword we split the keyword phrase into individual words and create a query that searches within the Wikipedia page titles requiring all the individual words to be found.

- Type 2: We split all keywords into individual words and combine those into one query searching within the Wikipedia page titles to find sentences on those pages where as many words as possible match the title of the page.

- Type 3: We combine all keywords as phrases into one query searching within the sentences to find those sentences where as many keywords as possible match.

We limit the number of results to the two most relevant sentences for the first query type and 20 sentences for the other two queries because the first query type makes one query per keyword while the other two only make one query per claim. An example of the queries being generated is given in Table 3. If the same sentence is found twice we do not add it to the candidate list again. For each of the candidate sentences we add the Wikipedia page title at the beginning of the sentence if it does not already contain it somewhere.

\subsection{Making the Prediction}

The second part of our system first processes the candidate sentences in three independent steps that can be run in parallel:

- We use a modified version of the Decomposable Attention neural network (Parikh et al., 


\begin{tabular}{llll}
$\#$ & Named Entity Recognition & Constituency Parser & Dependency Parser \\
\hline 1 & Northern Isles, Scotland & The Northern Isles & The Northern Isles \\
2 & - & Artificial intelligence, concern & Artificial intelligence, concern \\
3 & Walk of Life & album, the highest grossing album & -
\end{tabular}

Table 1: Generated keywords from the three systems (see Table 2 for claims). For the first claim the NE recognition correctly finds the two named entities while the other two systems miss one entity and got an additional The into the keyword. The second claim has no named entities and the other systems correctly find the relevant parts. In the third example the named entity found by the NE recognition is disambiguated by the Constituency Parser.

\begin{tabular}{|c|l|}
\hline$\#$ & Claim \\
\hline 1 & The Northern Isles belong to Scotland. \\
2 & Artificial intelligence raises concern. \\
3 & Walk of Life (album) is the highest grossing album. \\
\hline
\end{tabular}

Table 2: Example claims used in tables 1/3.

2016) to predict the textual entailment between each candidate sentence and its corresponding claim.

- We merge all candidate sentences of a claim into one block of text and predict the textual entailment between this block of text and the claim.

- We assign points to each candidate sentence based on POS-Tags.

Finally our system combines the results in order to decide on the label and to predict the evidence sentences for each claim.

\section{Textual Entailment}

We started with the Decomposable Attention network (Parikh et al., 2016) that is also used in the baseline except that we predict the textual entailment for each pair of candidate sentence and claim. We found that for long sentences the network has high attention in different parts of the sentence that semantically belong to different statements. Using the idea that long sentences often contain multiple statements we made the following additions to the Decomposable Attention network.

We include an additional 2-dimensional convolution layer that operates on the attention matrix in case of sufficiently large sentences. Based on our testing we decided on a single convolution layer with a kernel size of 12 . The output of this convolution layer contains a different amount of elements depending on the size of the attention matrix. This makes sense as longer sentences can contain multiple statements. We use a CnnEn- coder $^{4}$ to change the different length output into a same length output. This is necessary in order to use the result of the convolution layer in a later step of the network and can be seen as a selection of the correct statement from the available data. The output of the CnnEncoder is concatenated to the input of the aggregate step of the network. If either the claim or the candidate sentence are shorter than 12 then we skip this additional step and concatenate a zero vector instead.

When predicting the textual entailment we do not reduce the probabilities to a final label immediately but keep working with the probabilities in the final prediction (see Section Final Prediction).

\section{Merge Sentences}

For each claim we merge all the candidate sentences into one block of text similarly to the baseline. We predict the textual entailment using our modified decomposable attention network. We found that the REFUTES label is predicted with very high accuracy. However, this is not the case for the other two labels. By including the results of this step we can improve the predicted labels for the REFUTES label as shown in Table 5. Comparing that to the full result given in Table 4 we can see that about $29.3 \%$ of correct REFUTES predictions are due to this step.

\section{Creating POS-Tags and Assigning Points}

We use the Stanford POS-Tagger (Toutanova et al., 2003) to create POS-Tags for all candidate sentences and all claims. We found that the Stanford POS-Tagger only uses a single CPU core on our system so we wrote a script that splits the file containing all claim or candidate sentences into multiple files. Then the script calls multiple POSTagger instances in parallel, one for each file. The results are then merged back into a single file.

\footnotetext{
${ }^{4}$ https://allenai.github.io/ allennlp-docs/api/allennlp.modules. seq2vec_encoders.html
} 


\begin{tabular}{llll} 
Query type & Query & Occurrence & Limit \\
\hline Type 1 & "Artificial" "intelligence" & must occur & 2 \\
Type 1 & "concern" & must occur & 2 \\
Type 2 & "Artificial" "intelligence" "concern" & should occur & 20 \\
Type 3 & "Artificial intelligence" "concern" & should occur & 20
\end{tabular}

Table 3: Generated queries for claim 2 (see Table 2). Claim 2 has two keywords where one contains two words. For the Type 1 query we create two queries where one query contains two separate words. For the Type 2 query we split all words and use them all in one query. For type 3 we omit the split and use entire keyword phrases as query.

\begin{tabular}{|l|lll|}
\hline & SUP & REF & NEI \\
\hline SUP & 3291 & 370 & 3005 \\
REF & 1000 & 3159 & 2507 \\
NEI & 1710 & 1142 & 3814 \\
\hline
\end{tabular}

Table 4: Confusion matrix of the full system prediction. Columns are predictions and rows the true labels.

\begin{tabular}{|l|lll|}
\hline & SUP & REF & NEI \\
\hline SUP & -8 & +52 & -44 \\
REF & -2 & +926 & -924 \\
NEI & -4 & +152 & -148 \\
\hline
\end{tabular}

Table 5: Confusion matrix change due to including the merge feature. Columns are predictions and rows the true labels.

Using the generated POS-Tags we assign scores to the candidate sentences. First each candidate sentence is assigned 5 different scores, one for each of the following POS-Tag categories: verbs, nouns, adjectives, adverbs and numbers. Each category score starts at 3 and is decreased by 1 for each word of the respective POS-Tag category that is in the claim but not in the candidate sentence. Duplicate words are considered only once. We do not allow the category scores to go negative. At the end the category scores are added together to create the final score which can be a maximum of 15.

\section{Final Prediction}

We create a matrix from the per candidate sentence textual entailment probabilities with the three labels as columns and one row per candidate. We reduce all three probabilities of a candidate sentence if it received 11 or less points. The number 11 is empirically determined using the development set. As shown in Figure 1 we are able to filter out most of the non-evidence sentences by looking only at candidate sentences whose point score is more than 11. Reducing the probabilities is done by multiplying them with 0.3 . This way they are always reduced below the minimum highest prob-

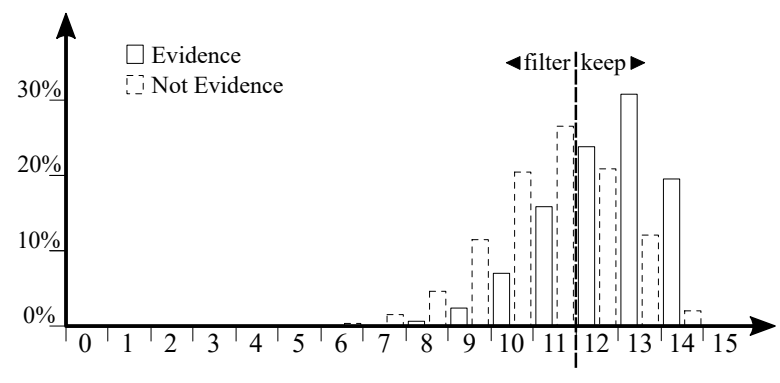

Figure 1: Histogram of how many candidate sentences (y-axis) received how many points (x-axis) for the development set. $65.07 \%$ of non-evidence and $26.21 \%$ of evidence sentences get filtered with the threshold between 11 and 12 .

ability of non-filtered sentences $(=33.33 \ldots \%)$.

Finally we predict the label and decide on the evidence sentences. If the Merge Sentences prediction predicted REFUTES then we use REFUTES as final label. Otherwise we find the highest value in the matrix and select the column it appears in as final label. We sort the matrix based on the column of the final label and select the top 5 candidate sentences as evidence.

\subsection{Training}

For training the modified Decomposable Attention network we are using the SNLI data set and the FEVER training set (Bowman et al., 2015; Thorne et al., 2018). For claims labeled as NOT ENOUGH $I N F O$ we first search for Wikipedia page titles that contain a word from the claim and then randomly choose one of the sentences on that page. If no Wikipedia page is found this way we randomly select one. We concatenate the generated training data with the SNLI data set to create the final training data containing 849,426 claims.

\section{Results}

Our system achieves a FEVER score of 0.3927 on the shared task development set containing 19, 998 


\begin{tabular}{|l|lll|}
\hline & Label & Recall & Score \\
\hline All & 0.5132 & 0.3581 & 0.3927 \\
Unmodified DA & 0.5170 & 0.3880 & 0.3909 \\
Without Points & 0.4545 & 0.1169 & 0.3665 \\
Without Merge & 0.4747 & 0.3294 & 0.3815 \\
\hline
\end{tabular}

Table 6: Contribution of each feature. Label refers to the label accuracy, while Recall refers to the evidence recall.

claims. This is a $25.5 \%$ improvement over the baseline score of 0.3127 on the development set. The confusion matrix for the predicted labels is given in Table 4. It shows that the highest incorrect predictions are for the NOT ENOUGH INFO label while the REFUTES label is predicted with the least amount of errors.

For the test set our system generated 773,862 pairs of candidate sentences and claim sentences. Only for a single claim out of all 19, 998 claims no candidate sentences were found.

For the development set the candidate sentences found in the first part of our system include the actual evidence of $77.83 \%$ of the claims. In comparison the baseline (Thorne et al., 2018) only finds $44.22 \%$ of the evidence. Our system finds 38.7 sentences per claim on average, while the baseline is limited to 5 sentences per claim.

When looking at how much each feature improves the final score in Table 6 , we can see that the point system using POS-Tags results in the biggest improvement.

\section{Conclusion}

In this paper we have presented our system for the FEVER Challenge. While keeping the two-part structure of the baseline we replaced the first part completely and heavily modified the second part to achieve a $25.5 \%$ FEVER score improvement over the baseline. In our immediate future work we will investigate alternative ways of obtaining higher recall in the first part but also improve the textual entailment to further reduce noise.

\section{References}

Samuel R. Bowman, Gabor Angeli, Christopher Potts, and Christopher D. Manning. 2015. A large annotated corpus for learning natural language inference. CoRR, abs/1508.05326.

Danqi Chen and Christopher Manning. 2014. A fast and accurate dependency parser using neural networks. In Proceedings of the 2014 Conference on Empirical Methods in Natural Language Processing
(EMNLP), pages 740-750. Association for Computational Linguistics.

Matt Gardner, Joel Grus, Mark Neumann, Oyvind Tafjord, Pradeep Dasigi, Nelson F. Liu, Matthew E. Peters, Michael Schmitz, and Luke Zettlemoyer. 2018. Allennlp: A deep semantic natural language processing platform. CoRR, abs/1803.07640.

Ankur P Parikh, Oscar Täckström, Dipanjan Das, and Jakob Uszkoreit. 2016. A decomposable attention model for natural language inference. arXiv preprint arXiv:1606.01933.

Matthew E. Peters, Mark Neumann, Mohit Iyyer, Matt Gardner, Christopher Clark, Kenton Lee, and Luke Zettlemoyer. 2018. Deep contextualized word representations. ArXiv e-prints, 1802.05365.

Mitchell Stern, Jacob Andreas, and Dan Klein. 2017. A minimal span-based neural constituency parser. CoRR, abs/1705.03919.

James Thorne, Andreas Vlachos, Christos Christodoulopoulos, and Arpit Mittal. 2018. FEVER: a large-scale dataset for fact extraction and verification. CoRR, abs/1803.05355.

Kristina Toutanova, Dan Klein, Christopher D. Manning, and Yoram Singer. 2003. Feature-rich part-ofspeech tagging with a cyclic dependency network. In Proceedings of the 2003 Human Language Technology Conference of the North American Chapter of the Association for Computational Linguistics. 